\title{
Cost Minimization of Aircraft Critical Components for Planning and Maintenance Requirements
}

\author{
Fatin Hamizah Abdol Rahim ${ }^{*}$, Norliza Mohd Noor ${ }^{2}$, Haslaile Abdullah', \\ Bayram Annanurov ${ }^{4}$
}

${ }^{1-3}$ Razak Faculty of Technology and Informatics, Universiti Teknologi Malaysia (UTM),

Jalan Sultan Yahaya Petra, Kuala Lumpur, 54100, MALAYSIA

${ }^{4}$ Paragon International University,

No. 8 St. 315, Boeng Kak 1, Tuol Kork, Phnom Penh, 12151, CAMBODIA

*Corresponding Author

DOI: https://doi.org/10.30880/ijie.2020.12.07.005

Received 12 March 2020; Accepted 3 August 2020; Available online 30 August 2020

\begin{abstract}
Spare parts management of the aircrafts is a part of maintenance planning that requires effective and efficient planning in order to reduce aircraft downtime in maintenance processes. Spare parts unavailability during maintenance is one of the factors that affects operational availability of the aircrafts fleet. This study focused on identifying the current problem affecting operational availability of the aircrafts fleet, developing an optimization model for cost minimization of spare parts and assessing the impact on spare parts availability using the cost minimization optimization model. Data analysis using machine learning techniques in WEKA was used to identify the current problem that is affecting aircrafts fleet operational availability. Then, Linear Integer Programming (LIP) and Goal Programming (GP) methods were used to develop the cost minimization optimization model by considering the current stock level of the critical components and the budget constrain. From this study, we discovered that spare parts unavailability during maintenance was the biggest contributing factor to the operational availability of the aircrafts fleet. Besides that, the cost minimization optimization model developed in the research had produced optimum level of inventory for the critical components with minimum cost. Spare parts availability for the aircrafts fleet had been improved with the application of the model which directly maximizes the operational availability of the aircraft fleet.
\end{abstract}

Keywords: Cost Minimization, Goal Programming (GP), Linear Integer Programming (LIP), Linear Regression, Machine Learning, WEKA

\section{Introduction}

Operational availability of aircrafts fleets in the military aviation is important to ensure readiness for operations, missions and trainings in safeguarding the security and sovereignty of the country in peace and war. Operational availability is defined as the ability of the aircraft to be deployed for the mission required [1]. In order to accomplish maximum operational availability, maximum number of aircrafts must be available to cope with the operations, missions and training requirements. Aircraft maintenance is necessary in order to maintain the safety, operational capability and airworthiness characteristics of the aircrafts. These maintenance activities can be divided into two categories. Firstly, the scheduled maintenance, which is to be conducted periodically after a certain flying hours or predicted period of time and secondly, is the unscheduled maintenance which is conducted when the aircraft is declared unserviceable due to any systems or components failure [2], [3], [4]. 
Aircraft maintenance planning involves a range of activities including management of materials (spare parts) and resources (labor, machine and equipment) [5]. The most critical planning is the maintenance planning and spare parts planning because unavailability of spare parts will cause delay in maintenance which will contribute to longer down time of the aircraft and high Mean Turn-around Time (MTAT). It important that both planning is optimized and integrated in order to reduce aircraft down time. Due to the complexity of the aircraft systems, the purchase, repair and maintenance of its components are usually associated with high cost and long lead time because the Original Equipment Manufacturer (OEM) or Maintenance Repair and Overhaul (MRO) agencies are from inside or outside of the country.

As military trainings are essential for military personnel, the availability of aircrafts for training plays an important role to ensure training requirements are met timely. As the aircraft maintenance consist of spare parts, maintenance and human resources planning, optimizing the plans will contribute to the achievement of maximum operational availability of the aircrafts fleet. Lack of spare parts will cause delays in aircraft's maintenance process, contribute to high MTAT and will affect its operational availability. Not only management of spare parts have high cost and long lead time, but it also depends on government's budget allocations. Therefore, with the budget constraints, spare parts planning must be optimized to achieve the optimum level of spare parts availability and maximize availability of the aircrafts fleet in order to keep up with the increasing training requirements while minimizing the cost.

The aim of this paper is to identify current problems affecting the operational availability of the aircrafts fleet, to develop an optimization model for cost minimization of spare parts and to assess the impact on spare parts availability in the application of the cost minimization optimization model. Documents review and data collection from the aircraft fleet and data analysis using machine learning techniques in WEKA were used to identify the current problem affecting the aircrafts fleet operational availability. Then, Linear Integer Programming (LIP) and Goal Programming (GP) methods were used to develop the cost minimization optimization model by considering minimum requirement by the aircrafts fleet, current stock level of the critical components and the budget constrain. This model will then be formulated by using Microsoft Excel and solved by using Excel Solver in order to produce an optimum inventory level with minimal cost.

\section{Related Work}

The current related work about aircrafts spare parts optimization is mainly about the optimizing the inventory stock level of spare parts in order to reduce the downtime of the aircraft due to unavailability of spare parts. Several approaches have been studied by authors in the past in order to achieve optimal stock level of spare parts, associating it with cost reduction. Previous related works were studied to understand the approaches used by the authors in order to construct the cost minimization optimization model.

One of the approaches is to allocate enough spare parts in various location of warehouse and operation stations. This approach had been implemented in both military and commercial airlines. For example, mathematical model METRIC (Multi-Echelon Technique for Recoverable Item) first introduced by Sherbrooke [6], [7] has been implemented by F. Costantino et al. with the example data from the Italian Air Force. It aims to minimize expected backorders in order to ensure availability of $99 \%$ of spare parts based on the actual flight plan. This study used marginal analysis to determine inventory stock level for each spare part of each warehouse by solving the mathematical model in order to fit the budget constraint and to achieve targeted operational availability by the Italian Air Force [8]. The same approach has been applied by Lei and Hongfu on the commercial aircraft [9] and Huimin Tang on flight university environment for determining the optimal inventory stock of Line Replaceable Unit (LRU) in order to reduce the backorders for critical LRUs [10]. This approach is implemented by the authors with the objective to allocate optimal stock level for various warehouses and operation stations in order to minimize the shipment cost between the locations in the event of stock out that requires the spare parts to be shipped from other locations.

Another approach is by predicting the demands of the failures spare parts in order to determine optimal stock level that is required to minimize the costs especially the loan and transportation costs. Probability Density Function (PDF) and Cumulative Density Function (CDF) have been used by J. Gu et al. in their research to predict number of failures of a spare part in the period of planning, where this predicted failures are used to as a basis to request the desired spare part in advance with the aim to minimize the inventory holding cost and also shortage costs [11]. Kirsten Tracht et al. uses the same approach in predicting the failure for Line Replaceable Unit (LRU) inventory stock level in the Maintenance, Repair and Overhaul (MRO) environment. Poisson distribution is used in this research to predict LRUs Failures and this prediction is used as a basis in determining the cost-optimal inventory level of the LRUs aiming to reduce the inventory holding and shortage costs [12].

There were several other studies that used the similar approach of estimating the future demands of spare parts in determining the inventory stock level such as H. Rezaei et al. where the reorder quantity is estimated by using the frequency usage of fast moving and slow moving items, which aims to minimize the total inventory cost while satisfying the targeted service level (TSL) [13] and also B. Brunaud et al. that predict the uncertainty demands of spare parts due to unscheduled maintenance which is used to determine the safety stock level of inventory with the aims to reduce stock out of spare parts [14]. Furthermore, Pleumpirom and Amorsawadwatana had developed a multi objective optimization model using goal programming in forecasting spare parts requirements for schedule maintenance with the aims to minimize cost, minimize lead time and maximize the quality of the spare parts. This model is used as a tool in making 
decision of either to purchase, repair or loan the spare parts for the scheduled maintenance requirements with the aims to reduce the total spare part cost, reduce maintenance down time and also improve the availability of the aircraft [15].

Based on literature review done for this study, spare parts availability is crucial in the aviation industry for both military and commercial airlines. Therefore, cost minimization in spare parts management can be achieved with good planning and decision making by the maintenance manager in the inventory management. This can be done by different approaches that available in the past literature. Improving spare parts availability not only minimize the cost of maintenance, but it will also improve operational availability of aircrafts fleet with better availability, reliability and maintainability of aircrafts [16].

\section{Research Method}

Data that is used in this study was collected from an aircraft fleet in Malaysia which was done by reviewing maintenance and defect trends records in order to identify the current problem affecting the operational availability of the aircrafts fleet. In order to ensure the reliability of the data used in this study, required data were collected starting from 2015 until 2018 and interviews were done if the data required were not recorded anywhere.

\subsection{Data Analysis Using Machine Learning}

The analysis on the data collected was conducted by using machine leaning software, WEKA in order to identify current problem that is affecting the aircrafts fleet. WEKA is a software tool that is equipped with various data mining techniques and algorithms that can be used in classifying and clustering data [17], [18], [19]. WEKA with linear regression classifier is used in this study in order to identify the relationship between parameters that were collected earlier.

Relation between a dependent variable with one or more independent variables can be measured and analyzed using regression [18], [20] and relationship between scalar variable with one or more variables can be modelled, by using a statistical approach of linear regression [18], [21]. Therefore, linear regression was used in this study to model the relationship between parameters that were collected from the aircraft earlier in order to identify the current problem that is affecting the operational availability of the aircrafts fleet.

Linear Regression model is build based on the data collected in the WEKA [17], [20], [22], [23] with the goal to predict the responses to $n$ data point $\left(x_{1}, y_{1}\right),\left(x_{2}, y_{2}\right), \ldots,\left(x_{n}, y_{n}\right)$ as Equation (1) below where $\beta_{0}$ and $\beta_{1}$ are the constants of the regression model and $x_{1}$ is the input variable [21]:

$$
y=\beta_{0}+\beta_{1} x_{1}
$$

Residual $\varepsilon_{i}$ at each of the $n$ data points are used to measure either the predicted value, $\beta_{0}+\beta_{1} x_{1}$ of the response variable $y$ fit the model that was built earlier as shown in Equation (2). Where in an ideal situation, where all the points lie on the model, all residuals $\varepsilon_{i}$ will be zero [21].

$$
E_{i}=y_{i}-\left(\beta_{0}+\beta_{1} x_{1}\right)
$$

However, in the real-life situation, it is impossible to obtain the ideal situation, therefore the residual will be minimized in obtaining the regression coefficients by using least square methods where the model constants will choose so that the sum of the squared residuals is minimized as Equation (3) below [21]:

$$
\text { Minimize } \sum_{i=1}^{n} E_{i}^{2}
$$

In this study, there are more than one input variables used to build the linear regression model, therefore Multiple Linear Regression as shown in Equation (4) below is used for the model. The parameters values of $\beta_{1}$ until $\beta_{n}$ are the weight for the input variables $x_{1}$ until $x_{n}$ and $\beta_{0}$ is the constant that will obtain from the linear regression model in order to minimize the sum of square of differences between actual and predicted $y$ values [21].

$$
y=\beta_{0}+\beta_{1} x_{1}+\beta_{2} x_{2}+\ldots+\beta_{n} x_{n}
$$




\subsubsection{Parameters}

Data analysis using WEKA with linear regression classification is conducted to identify the current problem that affecting the operational availability of the aircrafts fleet. Therefore, parameters regarding the performances of the aircrafts fleet are collected to be further analyzed using the WEKA software. Parameters that used in the analysis are as follows:

- Aircraft No. - represents the aircraft tail number for each aircraft in the aircrafts fleet.

- Hours Flown (Max. 342.86) - represents the hours flown by each aircraft where the maximum is 342.86 hours per year.

- Availability - represents the percentage availability for each aircraft.

- Availability (Hours) - represents the total hours of each aircraft is available.

- Downtime Hours - represents total downtime hours for each aircraft.

- AWP Hours - represents the total hours for each aircraft is waiting for spare parts during maintenance.

- Total Defect - represents the total systems failure for each aircraft.

During the pre-process of the data, Aircraft No. is removed from the input data in order to ensure all the data process in the linear regression are numeric. Availability and Availability (Hours) are also removed from the input data due to both parameters only represent the aircraft is serviceable and available for operational but not flown by the pilots. Therefore, Hours Flown (Max. 342.86) is used as the response variable in the linear regression model in order to predict how the other parameters affected the operational capability of the aircrafts fleet.

Analysis process of these parameters are shown in the Fig. 1 below. The input dataset containing the Downtime Hours, AWP Hours, Total Defect and Hours Flown (Max. 342.86) are used to build the linear regression model where the Hours Flown (Max. 342.86) is set as dependent variable. Based on the output that was obtained from the classifier, factors that affected the operational capability of the aircraft fleet can be determined henceforth determining the factors affecting the operational availability.

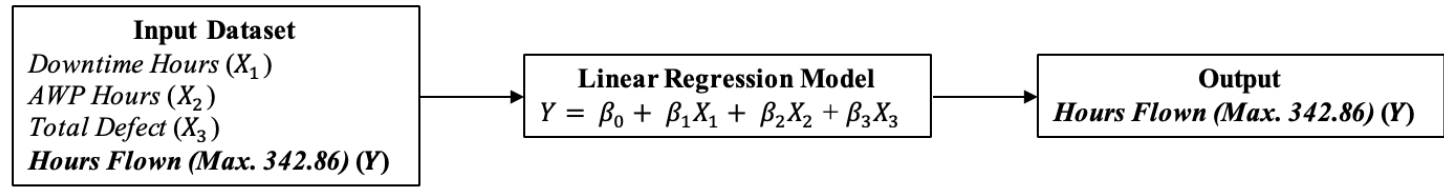

Fig. 1 - Multiple Linear Regression model

\subsection{Cost Minimization Optimization Model}

A few approaches had been reviewed earlier where mathematical programming was the most frequent method used by researchers because of the numerous variables involved in the optimization modelling [24]. Thus, Linear Integer Programming (LIP) and Goal Programming (GP) were used in this study because it fits the criteria where none of quadratic or nonlinear functions were included. These approaches are selected due to the high efficiency and flexibility of the modelling and also the availability of optimization software to solve the problems [25], [26].

Aircraft systems are very complex and require a lot of spares in maintenance. Based on the review of documents and discussion with the maintenance department, 60 critical components have been listed. These components are listed as critical due to long lead time and high cost. Therefore, the aircrafts fleet is required to maintain minimum stock level of these components in order to support the aircrafts fleet. However, there are some critical components with inadequate information that is removed from this paper. This study will focus on these 54 critical components of the aircrafts fleet.

Thus, unit cost, current stock inventory level and budget allocations will be collected in order to formulate the cost minimization optimization model. History of purchase and repair of these components will give a better understanding on the spare requirements for the critical components of the aircrafts fleet. Optimum spare parts inventory level with minimum cost will be the target for the cost minimization model to achieve in order to improve spare parts availability for the aircrafts fleet.

Developing the cost minimization model optimization model is the most critical part in this study. This model will be developed by using LIP and GP in the Microsoft Excel using Excel Solver based on data collected and the understanding of the aircrafts fleet requirements. This information will be implemented in order to prepare the cost minimization optimization model in the real world.

Cost minimization for critical components modelling process is a complicated process because there are a lot of factors need to be considered in order to achieve the best model for cost minimization. Therefore, mathematical models are used to generate optimized models. A general modelling framework of the whole process of cost minimization for critical components is required to describe the inputs and outputs of the model.

Input data for the critical component cost minimization requirements such as quantity per aircraft, minimum stock required, current inventory stock level, unit cost and budget allocations are identified in order to generate a practical cost 
minimization model. Besides that, history of purchase or repair of these critical components are also identified in order to predict the requirements of these critical components.

The expected output of the cost minimization optimization model should be able to display the minimum cost for the critical components together with its optimum stock level that is required for maintenance. The sets of inputs and outputs for the cost minimization optimization model is shown in Fig. 2 below.

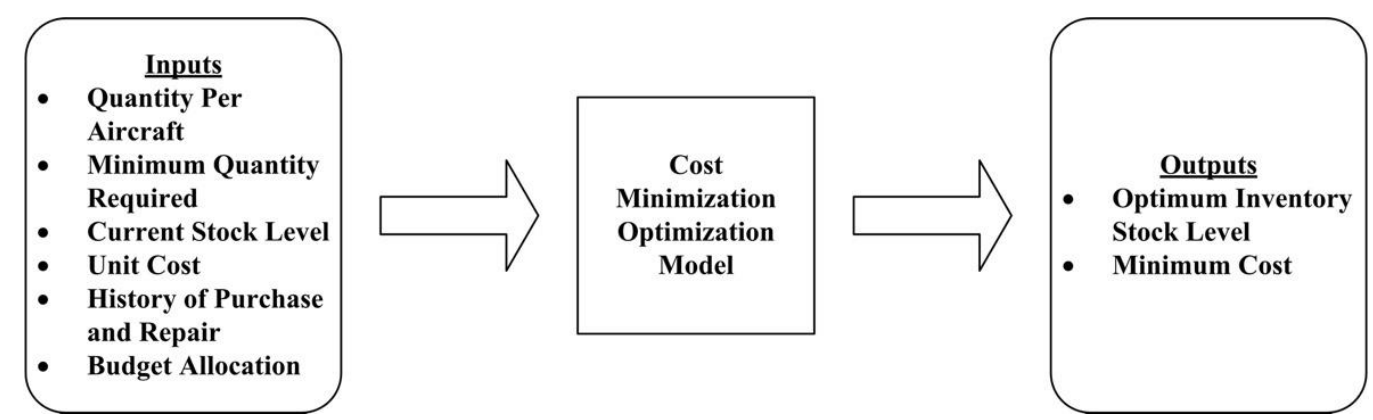

Fig. 2 - Modelling framework for cost minimization optimization model

\subsubsection{Linear Integer Programming (LIP)}

Based on the modelling framework, an equation for Linear Integer Programming (LIP) can be formulated to represent the cost minimization optimization model. The objective function of this model is represented in Equation (5) below:

$$
\text { MinimizeCost } Z=\sum_{j=1}^{n} \text { UnitCost } \times \text { Quantity Re quired }
$$

Total minimize cost is summation of the quantity required multiplied with the unit cost of the component. This can be represented by the mathematical Equation (6) as follows:

$$
\operatorname{Min} Z=\sum_{j=1}^{n} C_{X_{j}} X_{j}
$$

Where $C_{X_{j}}$ represents the component unit cost and $X_{j}$ represents the quantity of component that is required to be purchased or repaired. The objective function is bounded by the minimum quantity requirements, $M Q R_{X_{j}}$ of the components by the aircrafts fleet. Which quantity of required components that need to be purchased or repaired, $X_{j}$ should be more than or equal to the $M Q R_{X_{j}}$ deducted by the current stock level $C S L_{X_{j}}$ of the components as represents in the Equation (7) and (8). This constraint is to ensure that $X_{j}$ is at least achieve the $M Q R_{X_{j}}$.

\section{Quantity Re quired $\geq$ MinimumQuantity Re quired -CurrentStockLevel (7)}

$$
X_{j} \geq M Q R_{X_{j}}-C S L_{X_{j}}
$$

In order to ensure that the model is in the feasible, $X_{j}$ should always positive. Besides that, if the $C S L_{X_{j}}$ is greater than $M Q R_{X_{j}}$, that components are not required to be purchased or repaired, therefore $X_{j}$ will be equal to zero as represented in the Equation (9). This is to ensure the total cost is minimized and there will be no excessive spare parts purchased or repaired. $X_{j}$ should be integer because it is impossible to purchase or repair only half or in decimal point of repair as represented in Equation (10).

$$
\begin{aligned}
& X_{j} \geq 0(j=1,2,3, \ldots, n) \\
& X_{j}=\text { int }
\end{aligned}
$$


This model is used to achieve the minimum requirement of components by the aircraft fleet with the minimize cost, but it could not fit the budget constraints where the model becomes infeasible when budget constraint is fit into the model. Therefore, a new model by using GP to solve the budget constraint.

\subsubsection{Goal Programming}

Based on the previous model using the LIP, GP is used to solve multi-objective optimization to achieve two goals, that is, to achieve minimum quantity requirement of components; and to purchase or repair the components within allocated budget. Objective function in the GP is to minimize the total overachievement and underachievement deviations, $Q$ from the goals as Equation (11) below. Where, $Q$ is the sum of the deviations from all desired goals. The $w_{i}$ are non-negative constants representing relative weight to be assigned to the deviational $d_{i}^{-}$and $d_{i}^{+}$within priority level.

$$
\text { Minimize } Q=\sum_{i=1}^{n} w_{i}\left(d_{i}^{-}+d_{i}^{+}\right)
$$

The same constraint for the minimum quantity requirements, $M Q R_{X_{j}}$ of the components by the aircrafts fleet is used in the GP in order to achieve the first goal as represented in Equation (12) and (13). The underachievement, $d_{i}^{-}$and overachievement, $d_{i}^{+}$deviations are assigned for every component since it is required for every component to be able to achieve at least its minimum requirement. The underachievement, $d_{i}^{-}$and overachievement, $d_{i}^{+}$deviations are assigned with the weighted constants, $w_{i}$ based on the previous history of the purchasing and repairing of the component. Binary constants 1 is assigned on the underachievement if the spare part is required to be purchased or repaired and 0 if otherwise.

\section{Quantity Re quired $\geq$ MinimumQuantity Re quired -CurrentStockLevel}

$$
X_{j}+d_{i}^{-}+d_{i}^{+} \geq M Q R_{X_{j}}-C S L_{X_{j}}
$$

Meanwhile, in order to achieve second goal, another constraint is used to ensure total cost of the component purchase or repair does not exceed the total allocated budget as represented in the Equation (14) and (15). The underachievement, $d_{i}^{-}$and overachievement, $d_{i}^{+}$deviations are also assigned to this constraint together with the weighted constants, $w_{i}$ in order to ensure the total cost is within the allocated budget. Binary constant 1 is assigned on the overachievement since the total cost must be within the allocated budget and 0 if otherwise.

$$
\begin{gathered}
\sum_{j=1}^{n} \text { UnitCost } \times \text { Quantity Re quired }=\text { TotalBudget } \\
\sum_{j=1}^{n} C_{X_{j}} X_{j}+d_{i}^{-}+d_{i}^{+}=\text {TotalBudget }
\end{gathered}
$$

In this model, $X_{j}, d_{i}^{-}$and $d_{i}^{+}$should be always positive is in order to ensure this model is feasible. If the $C S L_{X_{j}}$ is greater than $M Q R_{X_{j}}$, the component is not required to be purchased or repaired, therefore $X_{j}$ will be equal to zero as in Equation (16). This is to ensure the total cost is minimize and there will be no excessive spare parts purchased or repaired. Again, $X_{j}$ should be integer because it is impossible to purchase or repair only half or in decimal point of repair as represented in Equation (17). Lastly, multiplication of $d_{i}^{-}$and $d_{i}^{+}$should be zero in order to ensure the underachievement and overachievement goal is achieved as represented in the Equation (18).

$$
\begin{aligned}
& X_{j}, d_{i}^{-}, d_{i}^{+} \geq 0(i, j=1,2,3, \ldots, n) \\
& X_{j}=\text { int }
\end{aligned}
$$




$$
d_{i}^{-} \times d_{i}^{+}=0
$$

The optimization models of the cost minimization of aircraft critical components will be developed and simulated in this study. LIP and GP of the cost minimization optimization models will be indicated and defined in the Microsoft Excel and solved by using Excel Solve, which will then simulate the results by using the sensitivity report in order to produce an optimum inventory level for the critical components while minimizing costs. Results obtained from both models will be compared with the existing data of inventory stock level and this process will be done until satisfactory results are obtained. The expected outcome of this paper is an optimum level of inventory stock level for the critical components with the minimized cost.

\section{Result and Analysis}

\subsection{Analysis Using WEKA}

Analysis is done using WEKA with the linear regression classification on the aircraft's performance data to identify the problem affecting aircrafts fleet operational availability. The analysis is conducted with data from 2016 until 2018 , considering the number of aircrafts that are available in the inventory since the aircrafts fleet only received additional new batch of aircrafts in 2016.

As stated earlier, output that is obtained from the linear regression classification is linear regression model that was built by from the input dataset that is loaded to the WEKA. Linear regression models obtained in this analysis are presented as the Equation (19), Equation (20) and Equation (21) for years 2016, 2017 and 2018 respectively. The other output on the correlation coefficient, means absolute error, relative absolute error, root relative squared error is in Table 1. Where $Y$ is the response variable of Hours Flown (Max. 342.86) and $X_{1}, X_{2}$ and $X_{3}$ are the coefficients of input variables which represent Downtime Hours, AWP Hours and Total Defect respectively. Surface plot for these models are shown in the Fig. 3 (a), (b) and (c) below.

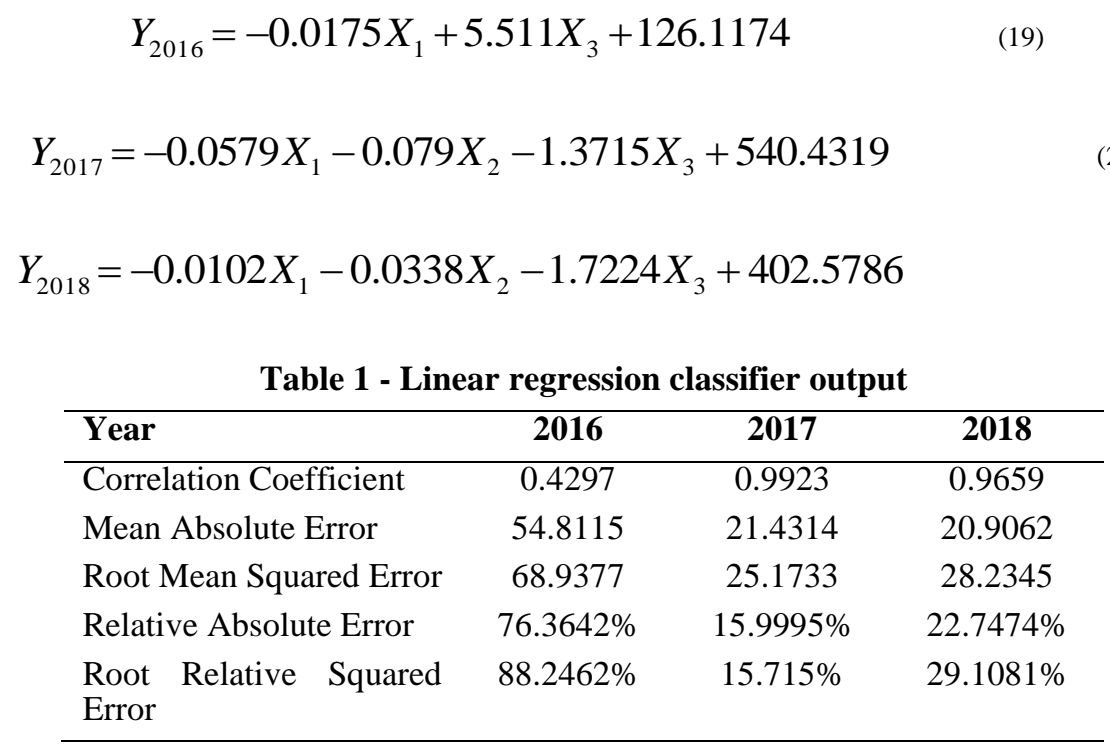




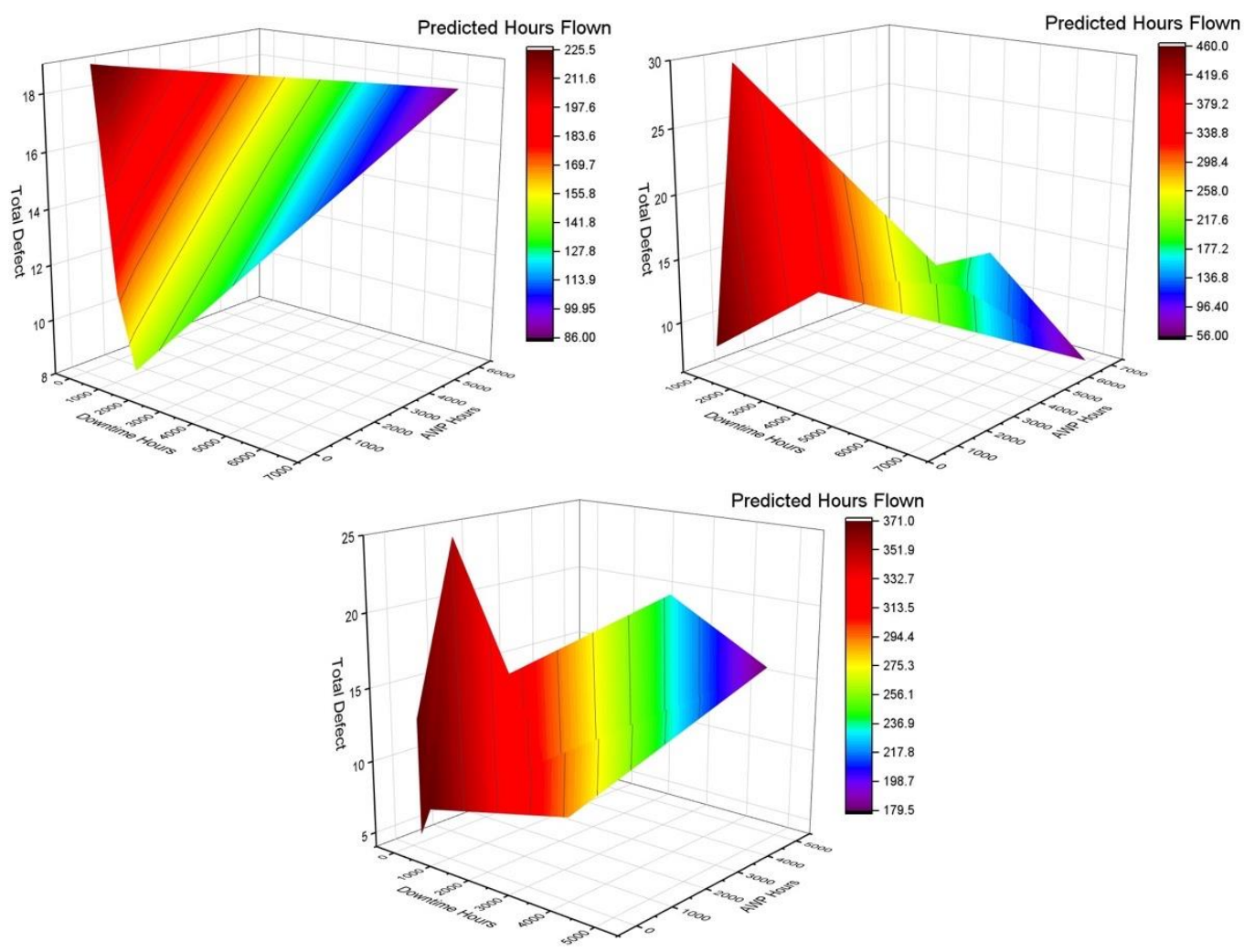

Fig. 3 - Aircrafts fleet data analysis for (a) 2016 (b) 2017 (c) 2018

Based on Equation (19) until (20), Downtime Hours and Total Defect are the parameters for building good model throughout the year of 2016 until 2018, while AWP Hours is only included in the year of 2017 and 2018. This is perhaps the best model that can be built from the data by WEKA. In the Table 1, the correlation coefficient implies the percentage of the variance in the data that explained by the model. Therefore, it shows that the data from the 2016 implies only $42.97 \%$ of the variance in the data that lies on the model while 2017 and 2018 implies $99.23 \%$ and $96.59 \%$ respectively.

Besides that, the mean absolute error (MAE) in the Table 1 is defined as total of absolute error that is divided with the number of predicted value and root mean square error (RMSE) is defined as the square root of the total of squares error divided by the number of predicted value, which both of these are used to measure the differences between the predicted value to the actual value [20]. Minimum values for both indicated the prediction and accuracy of the model is better. Hence, from the output obtained, models for 2017 and 2018 had better accuracy and prediction compared to 2016 but it is still the best model that can be built based on the data.

In these three models, Hours Flown (Max. 342.86) depends on the Downtime Hours, AWP Hours and Total Defect with the Total Defect carried more weight compared to the other two factors for all the three years which will be either positively or negatively affected the Hours Flown (Max. 342.86) of the aircraft fleet. Therefore, Total Defect contributing higher weightage on the factors that affecting Hours Flown (Max. 342.86).

Based on the result from the analysis, Total Defect is one of the major contributing factor that affected the operational availability of the aircrafts fleet as stated in the literature review [11], [12], [13], [14]. Therefore, it is crucial to focus on the spare parts availability in order to achieve higher hours flown which will improve the operational availability of the aircrafts fleet [11], [12], [13], [14], [27]. The availability of spare parts is trying to be achieved by implementing the cost minimization optimization model will be discussed in the next part of this paper.

Spare availability has been identified as the current problem that affected the operational availability of the aircrafts fleet through documentation review and further analysis on the data collected from the maintenance department. Therefore, in order to improve the spare availability cost minimization optimization models are proposed to achieve the optimum level of stock inventory level of spare parts with minimum cost. 


\subsection{Cost Minimization Optimization Model 4.2.1 Linear Integer Programming (LIP)}

As stated in the methodology earlier, the aircrafts fleet had listed 54 components as critical components for aircrafts fleet. These components are listed as critical component due to long lead time and high cost. Therefore, in order to formulate the cost minimization optimization models for the critical components, data on the current inventory stock level, unit cost and history of purchase or repair of the components are collected from the maintenance department.

Minimum requirement and the current stock level of the critical components are as shown in the Fig. 4. There are components that already fell below the minimum quantity requirements set by the management. Manager should consider this matter in making decision for purchasing or repairing the components. Therefore, this factor needs to be included in formulating the cost minimization optimization model in order to achieve the minimum cost in purchasing or repairing the components and to avoid excessive inventory holding.

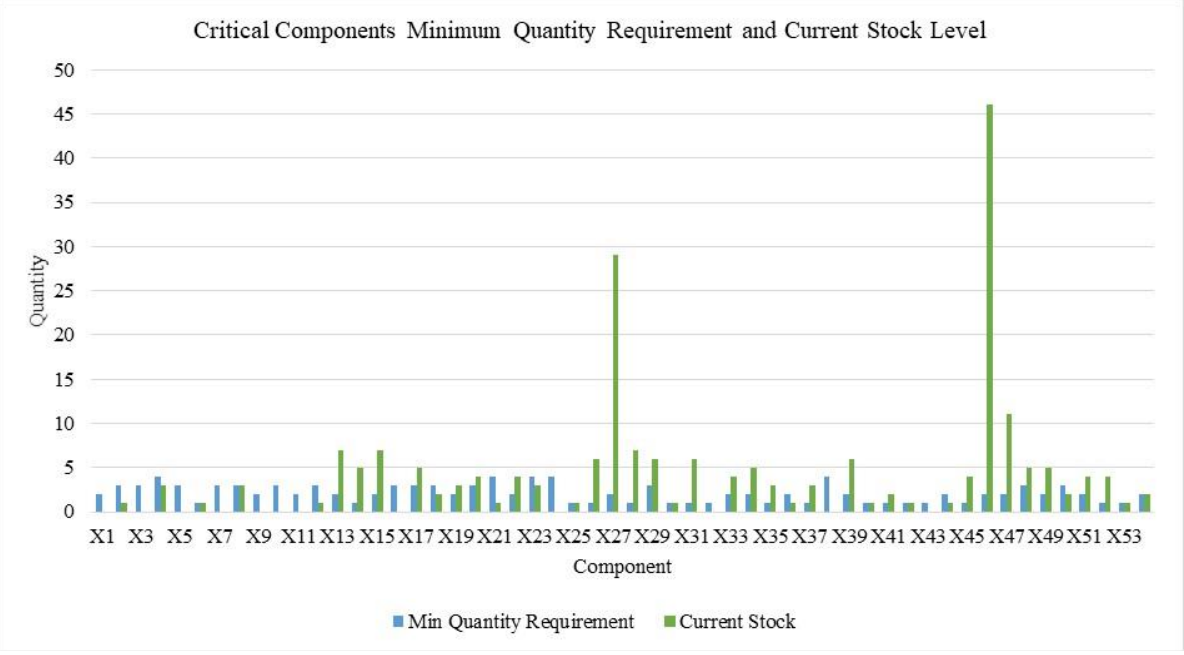

Fig. 4 - Critical components minimum quantity requirement and current stock level

Besides that, history of purchasing and repairing of components from the year of 2014 until 2018 are also collected and tabulated in Fig. 5. This factor needs to be considered in formulating the cost minimization optimization model in order to set the weightage in making the decision of purchasing and repairing the components. The higher the number of components purchased or repaired in the past means that the components are required more at the aircrafts fleet. Therefore, manager should set higher the priority for the component to be purchased or repaired in the future planning.

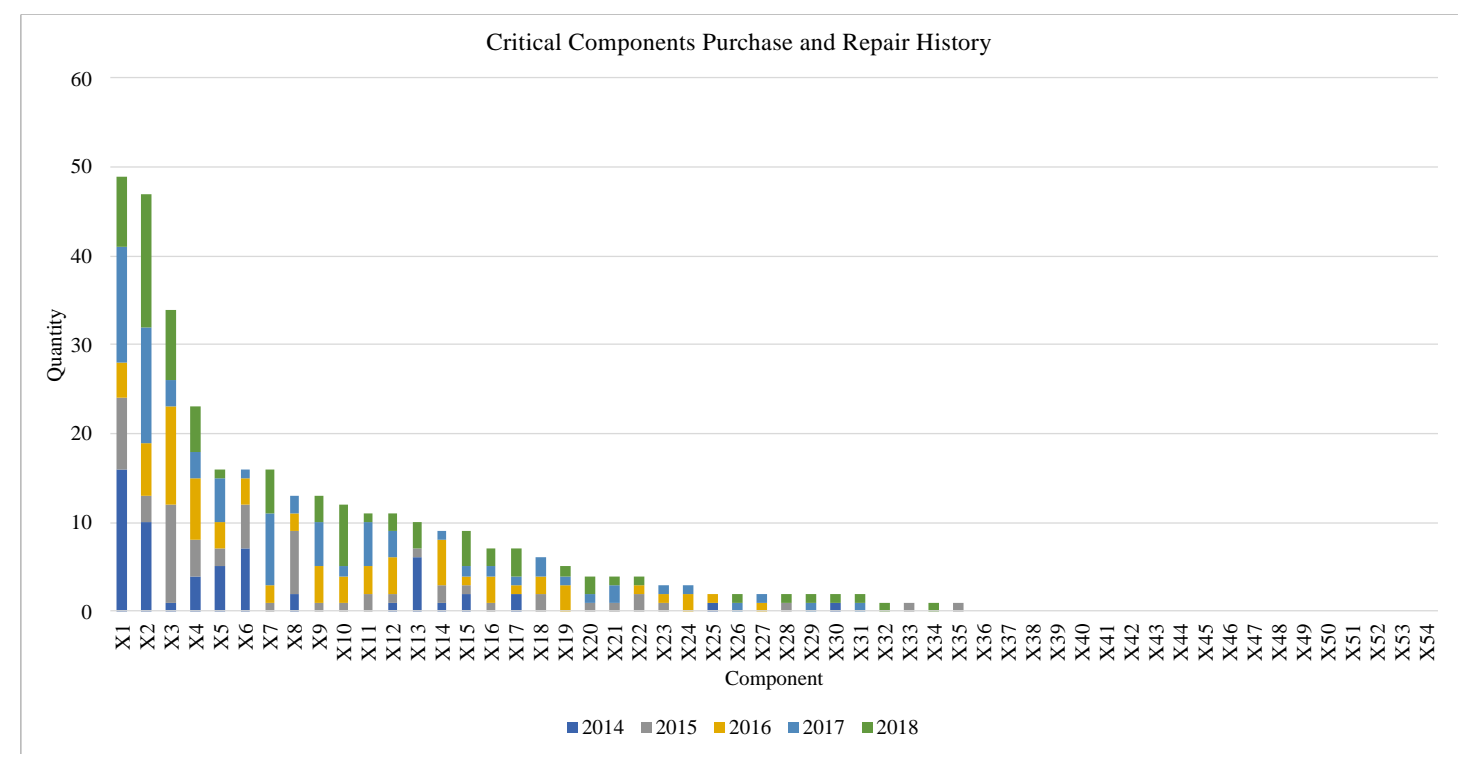

Fig. 5 - Critical components purchase and repair history 
Based on the critical components data that was collected from the maintenance department, a LIP mathematical model is formulated using Equation (5) until (10) using Microsoft Excel and solved using Excel Solver with the goal to minimize the cost, $Z$ and achieve at least the minimum quantity requirement of the critical components.

This model had considered the minimum quantity requirement and current stock level of the critical components. Where the component quantity required to be purchased or repaired are made by comparing the minimum requirement of the components and current stock level of the components. If the components exceed the minimum quantity requirement, that component will not be included in the solution in order to avoid the excessive stock holding and to minimize the cost of purchasing and repairing the critical components.

The result obtained from the LIP are shown in Fig. 6 (a) and (b). Implementation of cost minimization optimization model using the LIP had successfully achieved all the minimum quantity requirements of the critical components with the Minimize Cost, $Z=R M 2,701,551.60$. This result shows that for the manager to achieve at least the minimum requirement quantity of the critical components, total cost of RM 2,701,551.60 needs to be invested by the management. However, this is not ideal due to the limited budget allocation by the government.

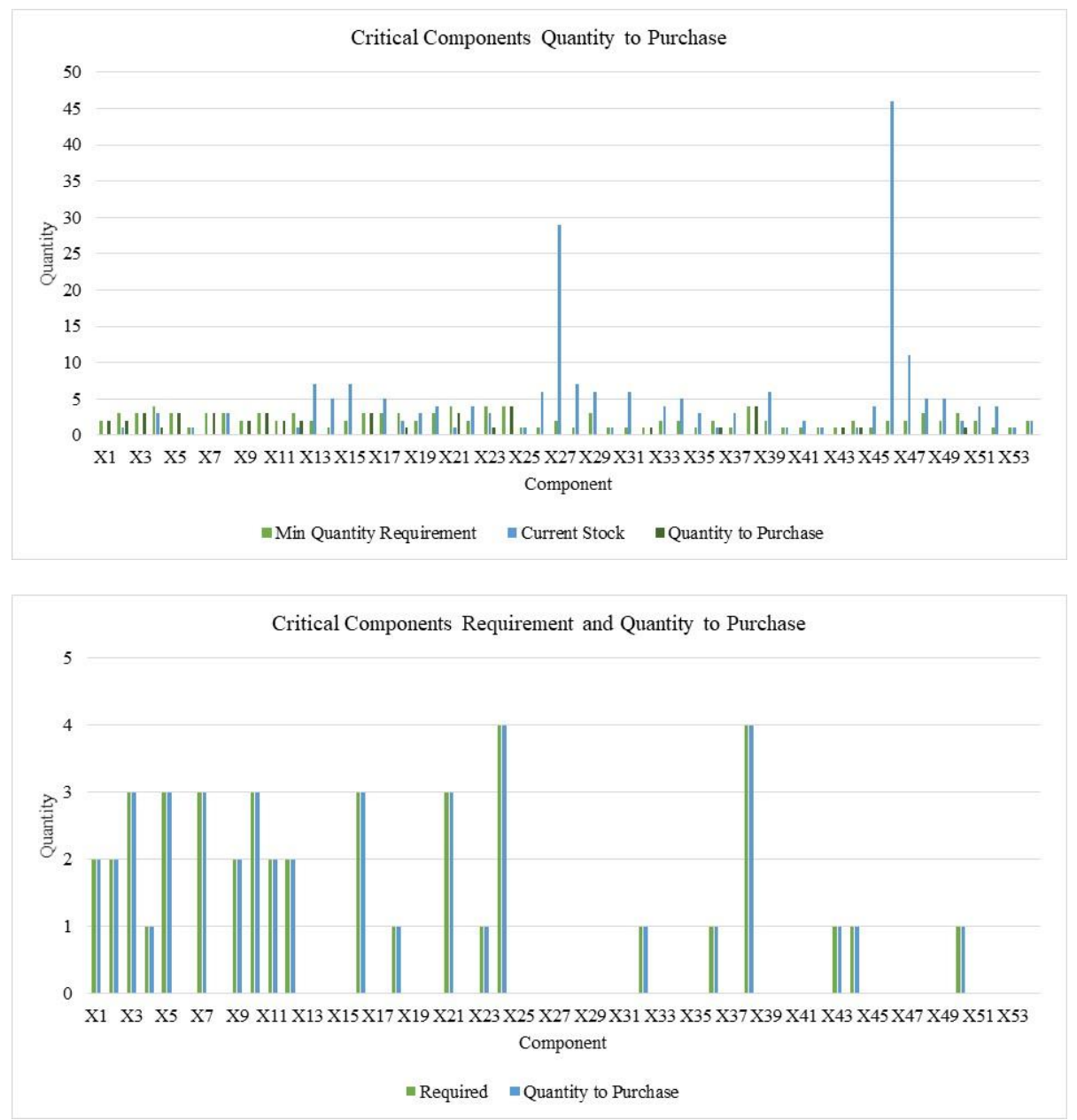

Fig. 6 - (a) Critical components quantity to purchase using LIP (b) Critical components requirements and quantity to purchase using LIP

Therefore, budget constraint needs to be fitted into the model in order to meet the actual budget constraint. However, when the budget constraint is fitted into the LIP model, the result obtained is infeasible due to the constraints of the model itself. The results are shown in the Fig. 7 (a) and (b), where the model could not achieve the minimum quantity requirement for all the critical components and the integer constraint is violated when the quantity required for components $X_{36}$ is set to be 0.4059 even the budget constraint is achieved at Minimize Cost, $Z=R M 2,000,000.00$. This shows that LIP can be used as a tool in decision making to purchase and repair the critical components only if there are no budget constraints.

Based on the literature review, spare parts inventory level that was achieved in the previous studies usually in the range of $95 \%$ to $99 \%$ of spare parts availability with the implementations of the model proposed in the studies [8], [14]. The result obtained from this model shows that the spare parts inventory stock level had obtained $100 \%$ of the minimum 
quantity requirement and this had improved the results that was obtained from the previous model. However, this model had produced infeasible solution when fitted with budget constraints and it shows that this model is not suitable to be implemented if there is limited budget allocation. This is not an ideal solution in the aviation industry since budget allocated for spare parts is usually limited due to the high operational cost in the industry.

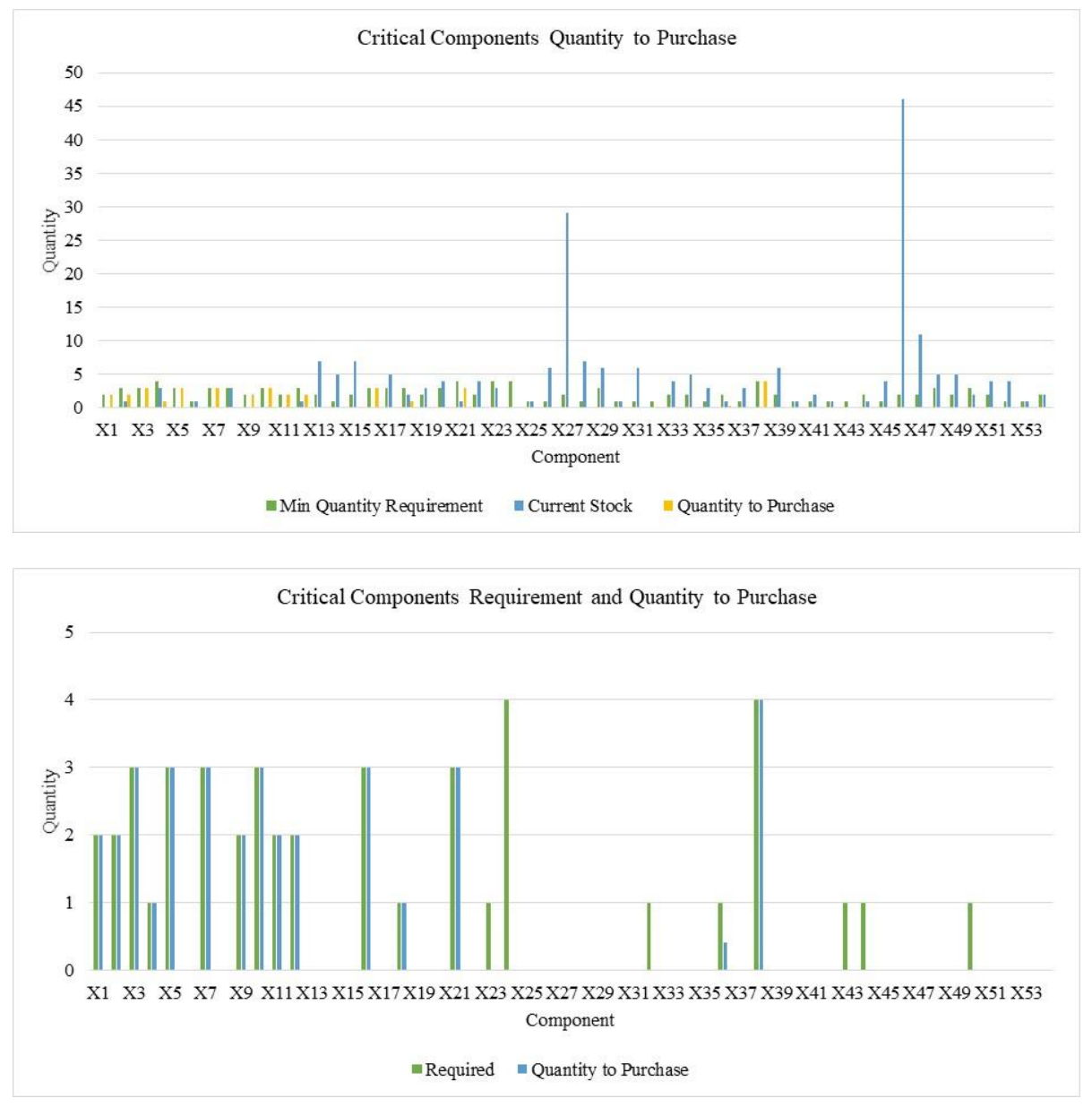

Fig. 7 - (a) Critical components quantity to purchase using LIP with budget constraint (b) Critical components requirements and quantity to purchase using LIP with budget constraint

\subsubsection{Goal Programming}

Due to limitation of Linear Integer Programming (LIP) in solving the budget constraint for the cost minimization optimization model for the critical components, Goal Programming (GP) is used to solve this constraint. The mathematical model is formulated using the Microsoft Excel and solved using Excel Solver with the goals to minimize the deviation, $Q$ and achieve at least minimum quantity requirement of the critical components within the budget allocated by the management.

Besides fitting the budget constraint into the model, this method also considers the history of purchasing or repairing the components data as shown in Fig. 5 where weightage binary constant is set to 1 if there is history of purchasing or repairing the component in the past from the year 2014 until 2018. This will put the priority to the component that had more history of defects in the past. The minimum quantity requirement and current stock level constraints are also included in this model in order to ensure there is no excessive stock inventory holding and to minimize the cost of purchasing and repairing the critical components.

The result obtained from the GP are shown in the Fig. 8 (a) and (b). Implementation of cost minimization optimization model using the GP had successfully minimized the deviation, Minimize $Q=0$ with the Total Cost $=R M 1,990,091.67$ required to achieve the optimal stock level of the critical components which is within the budget constraint although not all the critical components achieved its minimum quantity requirements. This total cost had reduced $26.34 \%$ from the minimize cost produced by the LIP earlier. 


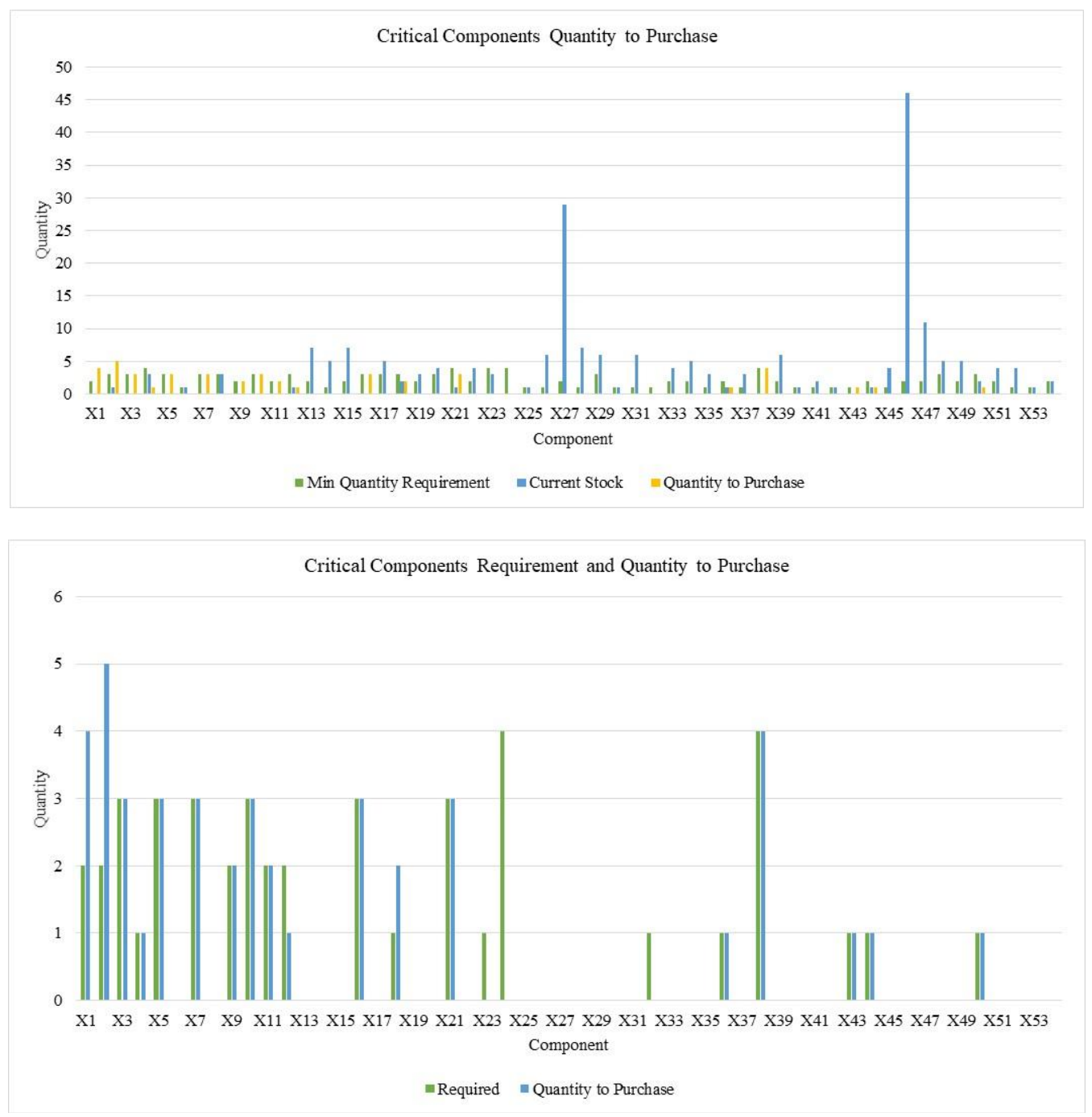

Fig. 8 - (a) Critical components quantity to purchase using GP (b) Critical components requirements and quantity to purchase using GP

Comparison between quantity to purchase or repair for the critical components produced by both programming models is shown in Fig. 9. Implementation of the LIP had obtained better result in achieving the minimum quantity requirement of the critical components than the GP. All the components $X_{1}$ until $X_{54}$ had successfully achieved their minimum quantity requirement with consideration of the current stock level. However, this is not an ideal solution for the aircrafts fleet since it will require more cost to be invested if there is limited budget allocated.

Therefore, implementation of GP method is more ideal for the aircrafts fleet although not all the minimum quantity requirement of the critical components achieved but this model had successfully fit the budget constraint and considered the current stock level and history of purchasing or repairing the critical components. Critical components $X_{1}$ and $X_{2}$ had overachieved its minimum quantity requirement and $X_{23}, X_{24}$ and $X_{32}$ had underachieved its minimum quantity requirement. This is because the GP had considered the historical data of purchasing and repairing the component, where $X_{1}$ and $X_{2}$ had higher history of purchasing and repairing compared to $X_{23}, X_{24}$ and $X_{32}$. Therefore, it is more optimal for the manager to choose overstocking the $X_{1}$ and $X_{2}$ in the planning. Components unit cost also contribute to this result, where the components unit cost of $X_{23}, X_{24}$ and $X_{32}$ is much higher than the $X_{1}$ and $X_{2}$ which will contribute to the violation of the budget constraint if the model choose to purchase or repair it.

Based on the literature review, total cost produced by the previous models is usually in the range $95 \%$ to $96 \%$ of total allocated budget [8], [12] and 95\% to $99 \%$ of spare parts availability [8], [14] with the implementations of the model proposed in the studies. Result from this model shows that $99.5 \%$ of allocated budget had been used in order to achieve $92.59 \%$ of the spare parts' minimum quantity requirement. This had improved the results that was obtained from the previous model in the term of using budget allocation but not in the terms of spare availability. Nonetheless, the results are considered as optimal solution considering the historical data on purchasing and repairing the spare parts. 


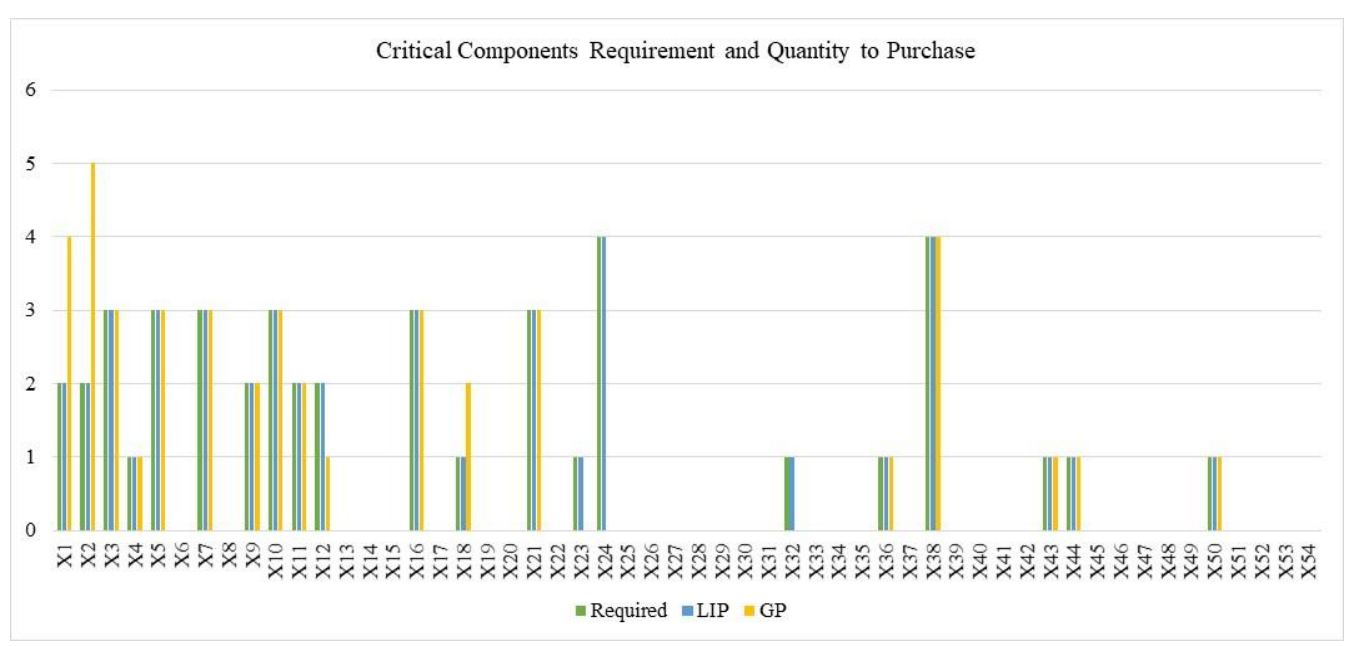

Fig. 9 - Critical components requirements and quantity to purchase using LIP and GP

\section{Conclusion}

As a conclusion, from the analysis and the result that was obtained from this study the current problem that was affecting the operational availability of the aircrafts fleet is identified due to the high downtime of the aircrafts due to waiting for spare parts in the maintenance process especially in the unscheduled maintenance. However, this defect trends are uncertainty and could not be predicted. Therefore, the aircrafts fleet needs to improve the spare parts availability in order to improve the operational availability of the aircrafts fleet.

However, due to the limited budget allocation by the government, the aircrafts fleet is required to minimize the cost in purchasing and repairing the aircrafts spare parts. Therefore, cost minimization optimization model that was developed in this study to be used as a tool by the maintenance manager in decision making for purchase or repair of spare parts. Implementation of the model using the GP is more suitable with the aircrafts fleet current situations with the consideration of limited budget allocation. However, implementation of the model using the LIP could also be used by the aircrafts fleet if the budget is not limited.

\section{Acknowledgement}

The authors would like to acknowledge various people and aircrafts fleet for their contributions to the analysis reported in this paper.

\section{References}

[1] Raju, V. R. S., Gandhi, O. P., \& Deshmukh, S. G. (2012). Maintenance, Repair, and Overhaul Performance Indicators for Military Aircraft. Defence Science Journal, 62(2), 83-89

[2] Kozanidis, G., Gavranis, A., \& Liberopoulos, G. (2014). Heuristics for flight and maintenance planning of mission aircraft. Annals of Operations Research, 221(1), 211-238

[3] Verhoeff, M., Verhagen, W. J. C., \& Curran, R. (2015). Maximizing Operational Readiness in Military Aviation by Optimizing Flight and Maintenance Planning. Transportation Research Procedia, 10, 941-950

[4] Gavranis, A., \& Kozanidis, G. (2015). An exact solution algorithm for maximizing the fleet availability of a unit of aircraft subject to flight and maintenance requirements. European Journal of Operational Research, 242(2), 631-643

[5] Samaranayake, P. (2006, 17-20 December 2006). Current Practices and Problem Areas in Aircraft Maintenance Planning and Scheduling - Interfaced/Integrated System Perspective. Paper presented at the Proceedings of the 7th Asia Pacific Industrial Engineering and Management Systems Conference 2006, Bangkok, Thailand

[6] Sherbrooke, C. C. (1968). Metric: A Multi-Echelon Technique for Recoverable Item Control. Operations Research, $16(1), 122-141$

[7] Sherbrooke, C. C. (1986). VARI-METRIC: Improved Approximations for Multi-Indenture, Multi-Echelon Availability Models. Operations Research, 34(2), 311-319

[8] Costantino, F., Di Gravio, G., \& Tronci, M. (2013). Multi-echelon, multi-indenture spare parts inventory control subject to system availability and budget constraints. Reliability Engineering \& System Safety, 119, 95-101

[9] Lei, S., \& Hongfu, Z. (2010, 26-28 May 2010). Multi-echelon inventory optimal model of civil aircraft spare parts. Paper presented at the 2010 Chinese Control and Decision Conference 
[10] Tang, H. (2017). Allocating Aircraft Repairable Spare Parts Inventory for Single Training Base of a Flight University. Paper presented at the 7th International Conference on Management, Education, Information and Control (MEICI 2017)

[11] Gu, J. Y., Zhang, G. Q., \& Li, K. W. (2015). Efficient aircraft spare parts inventory management under demand uncertainty. Journal of Air Transport Management, 42, 101-109

[12] Tracht, K., von der Hagen, F., \& Schneider, D. (2013). Applied repairable-item inventory modeling in the aviation industry. In R. Roy, A. Tiwari, A. Shaw, C. Bell, \& P. Phillips (Eds.), 2nd International through-Life Engineering Services Conference (Vol. 11, pp. 334-339)

[13] Rezaei, H., Baboli, A., Shahzad, M. K., \& Tonadre, R. (2018). A new methodology to optimize target stock level for unpredictable demand of spare parts: A Case Study in Business Aircrafts' Industry. IFAC-PapersOnLine, 51(11), $538-543$

[14] Brunaud, B., Laínez-Aguirre, J. M., Pinto, J. M., \& Grossmann, I. E. (2019). Inventory policies and safety stock optimization for supply chain planning. AIChE Journal, 65(1), 99-112

[15] Pleumpirom, Y., \& Amornsawadwatana, S. (2012). Multiobjective optimization of aircraft maintenance in Thailand using goal programming: A decision-support model. Advances in Decision Sciences, 2012

[16] Ghobbar, A. A., \& Friend, C. H. (2004). The material requirements planning system for aircraft maintenance and inventory control: a note. Journal of Air Transport Management, 10(3), 217-221

[17] Hall, M., Frank, E., Holmes, G., Pfahringer, B., Reutemann, P., \& Witten, I. H. (2009). The WEKA data mining software: an update. SIGKDD Explor. Newsl., 11(1), 10-18

[18] Novaliendry, D., Hendriyani, Y., Yang, C. H., \& Hamimi, H. (2015). The optimized K-means clustering algorithms to analyzed the budget revenue expenditure in Padang. Paper presented at the International Conference on Electrical Engineering, Computer Science and Informatics (EECSI)

[19] Özdemir, A., Yavuz, U., \& Dael, F. A. (2019). Performance evaluation of different classification techniques using different datasets. International Journal of Electrical and Computer Engineering, 9(5), 3584-3590

[20] Sahoo, G., \& Kumar, Y. (2012). Analysis of parametric \& non parametric classifiers for classification technique using WEKA. International Journal of Information Technology and Computer Science (IJTCS), 4(7), 43

[21] Mihăescu, M. C. (2011, 18-21 Sept. 2011). Classification of learners using linear regression. Paper presented at the 2011 Federated Conference on Computer Science and Information Systems (FedCSIS)

[22] Holmes, G., Donkin, A., \& Witten, I. H. (1994). WEKA: a machine learning workbench. Retrieved from https://hdl.handle.net/10289/1138

[23] Witten, I. H., Frank, E., Trigg, L. E., Hall, M. A., Holmes, G., \& Cunningham, S. J. (1999). Weka: Practical machine learning tools and techniques with Java implementations. Retrieved from https://hdl.handle.net/10289/1040

[24] Bergh, J. V. d., Bruecker, P. D., Beliën, J., \& Peeters, J. (2013). Aircraft Maintenance Operations: State of Art. FEB@HUBRUSSEL RESEARCH PAPER(Nr. 2013/09)

[25] Nemati, M., Braun, M., \& Tenbohlen, S. (2018). Optimization of unit commitment and economic dispatch in microgrids based on genetic algorithm and mixed integer linear programming. Applied Energy, 210, 944-963

[26] Yin, J., Yang, L., Tang, T., Gao, Z., \& Ran, B. (2017). Dynamic passenger demand oriented metro train scheduling with energy-efficiency and waiting time minimization: Mixed-integer linear programming approaches. Transportation Research Part B: Methodological, 97, 182-213

[27] Aisyati, A., Jauhari, W. A., \& Rosyidi, C. N. (2013). Determination Inventory Level for Aircraft Spare Parts Using Continuous Review Model. International Journal of Business Research and Management (IJBRM), 4(1), 1-12 